\title{
Toxic Shock Syndrome Without a Retained Tampon
}

\author{
Olivia Nagle ${ }^{1}$, David D Souza ${ }^{2 *}$ and Tom Shortland ${ }^{3}$ \\ ${ }^{1}$ Buckingham University, Medical Student, United Kingdom \\ ${ }^{2}$ Consultant \& Honorary Associate Professor Obstetrics \& Gynecology, South Warwickshire Foundation Trust, Warwick, \\ United Kingdom
}

${ }^{3}$ Warwick University, Medical Student, United Kingdom

*Corresponding author: David D Souza, Consultant \& Honorary Associate Professor Obstetrics \& Gynaecology, South Warwickshire Foundation Trust, Warwick, United Kingdom

\begin{tabular}{|c|c|}
\hline ARTICLE INFO & ABSTRACT \\
\hline Received: August 13, 2019 & Keywords: Toxic Shock; Retained Tampon; Enterotoxins G \& I; Staphylococcus Aureus \\
\hline Published: 㓞 August 22, 2019 & $\begin{array}{l}\text { Abbreviations: TSS: Toxic Shock Syndrome; ICU: Intensive Care Unit; GCS: Glasgow } \\
\text { Coma Scale; PID: Pelvic Inflammatory Disease; TSST: Toxin Shock Syndrome Toxin }\end{array}$ \\
\hline
\end{tabular}

Citation: Olivia Nagle, David D Souza, Tom Shortland. Toxic Shock Syndrome Without a Retained Tampon. Biomed J Sci \& Tech Res 20(5)-2019. BJSTR. MS.ID.003504.

\section{Introduction}

Toxic Shock Syndrome (TSS) is a rare and potentially fatal condition associated with multiple organ failure. Caused by a toxic strain of Staphylococcus Aureus and Streptococcus Pyogenes, TSS has historically been linked to the use of tampons in menstruating women and often associated with them being either "misplaced or forgotten" (i.e. retained tampon). In the absence of a single diagnostic test, the diagnosis is often based on the clinical presentation of the patient, but sometimes the patient can be so moribund, that a full history is difficult to obtain. A wider understanding of other potential gynaecological causes and atypical presentations can prompt physicians to initiate rapid antibiotic therapy before a full diagnosis is established. I report a case of Toxic Shock Syndrome in the absence of a "retained tampon", caused by enterotoxins G \& I released by a Staphylococcus Aureus infection.

\section{Case Report}

A 16-year-old nulliparous Caucasian female presented to the Accident \& Emergency department with a history of a "widespread rash" all over her body, vomiting and fever for the previous two days. Further questioning revealed that she had neck stiffness and it was noted that she had recently finished her menstruation. With the initial diagnosis of sepsis secondary to meningitis, she was commenced on treatment with Acyclovir, Clindamycin and
Ceftriaxone. Although the patient was initially alert, she became unconscious and was subsequently admitted to Intensive Care Unit (ICU), intubated and ventilated due severe hypotension, with a systolic blood pressure of 50-60mmhg, and a fluctuating Glasgow Coma Scale (GCS). Noradrenaline, adrenaline and vasopressin were used to maintain her cardiovascular function, whilst IV steroids were administered due to the suspicion of meningitis. As the patient was unconscious and fully intubated and did not appear to be responding to the antibiotics or antiretrovirals, further expertise from other specialities was sought. In view of her recent history of finishing her menstruation, the gynaecologists were asked to review her in ICU. As she was intubated, the full history with sensitive questions were obtained through the parents of the 16 year old. She had no significant past medical history of note and her periods were regular (28/5) and not particularly heavy or painful. Her mother was aware that she had just recently finished her period and recognised the need to remove the tampons after less than 8 hours. She thought that her daughter had never been sexually active and had no history of pelvic inflammatory disease (PID).

She was noted to have bilateral conjunctival discharge as well as a history of vaginal discharge. The intensivists called for repeat gynaecological examinations due to their concerns about the possibility of toxic shock from a "retained tampon", but on each 
of the three times that she was examined by a separate senior gynaecologist, no tampon was visible and only minimal discharge was apparent. A high vaginal and endocervical swabs were taken to exclude any specific pelvic infection, but the consensus was that there was no obvious gynaecological cause of her symptoms. The Beta-HCG was negative and throat swabs showed no evidence of Herpes Simplex Virus. An abdominal ultrasound again confirmed no abnormalities within the abdomen or pelvis and no signs of PID. There was a small pelvic collection of fluid suggestive of blood and a small pleural effusion in the left lung, but nil else of note. The gynaecological team were very reluctant carrying out any further investigations although did discuss the possibility of laparoscopy +/- suction irrigation of a fluid in her pelvis if her symptoms continued or worsened.

The cardiology team were closely involved in the multidisciplinary approach, dueto suspected septic cardiomyopathy (LV impairment: ejection fraction 10\%). The patient was also reviewed by the paediatric team. Due to a severely deranged clotting profile (Platelets 43, INR 2.6) and low likelihood of significant diagnostic benefit, a lumbar puncture was not performed. PCR testing for meningococcal disease and pneumococcal disease were negative and she was given a platelet transfusion to restore her clotting profile following a discussion with the haematology team. Blood cultures and vaginal swabs which were sent at her initial presentation, came back showing evidence of Staphylococcus Aureus and these were positive for Enterotoxin $\mathrm{G}$ and I after being sent to Public Health England Laboratory for verification. A diagnosis of Toxic Shock Syndrome was made, despite the absence of a retained tampon.

She was commenced on Meropenem immediately on the advice of the microbiologist and the other medications were ceased. On her fourth day on ICU, she was extubated but desaturated and became "very agitated" resulting in her having to be sedated again and re-intubated with ongoing respiratory support. Following further vasopressors and diuretic treatment, she was successful extubated on day seven. The patient completed her course of Meropenem and was transferred to the paediatric ward. Her left ventricular function returned to normal and the left sided pleural effusion resolved. Twelve days after her initial admission to hospital, she was discharged with a follow up with cardiology. From the gynaecological perspective she was simply advised to use pads rather than tampons in the future.

\section{Discussion}

With an average annual incidence of $0.07 / 100,000$ in the UK, TSS clinically presents with fever, hypotension and a rash (CDC, 1980). It is caused by the immune activation by superantigens: Staphylococcal Toxin Shock Syndrome Toxin (TSST-1), Streptococcal Toxic Shock Syndrome Toxin (STSS) as well as various other enterotoxins Pringle et al. [1]. Staphylococcus Aureus is a commensal bacterium commonly found in the nose and vagina of humans and may also present in soft tissue infections, burns and foreign bodies (e.g. retained nasal packaging) Ross \& Shoff [2]. The presence of Enterotoxin G and I leads to a definitive diagnosis of TSS. This "toxin mediated" disease results in an uncontrolled cytokine proliferation and overactivation of inflammatory cells, resulting in multisystemic organ failure.

The cause of TSS can be divided into two categories: nonmenstrual and menstrual. The former can be caused by caesarean or postpartum vaginal infections whilst the latter is associated with "prolonged use" of an individual tampon and is the most commonly recognised cause by the public and healthcare professionals together. Over $45 \%$ of cases have been associated with menstruation, whilst the remainder are associated with nonmenstrual causes Reingold et al. [3]. "Menstrual" TSS is typically associated with the absorbency of tampons in menstruating women, making headlines in 1978 following the death of several women using "super-absorbent" tampons. The brand: Rely Tampons had generated a "medical crisis" and were subsequently withdrawn from the market in 1980, following a report from CDC linking them to the development of TSS. Whilst the majority of tampons are made from cotton and rayon, Rely Tampons were unique in that they used carboxymethylcellulose for absorption.

They were designed to absorb twenty times their own weight in fluid, but the vaginal dryness which developed was found to be a significant risk factor for bacteraemia +/- septicaemia MacPhee et al. [4]. Equally, the length of time a tampon is worn has spiked an increased public awareness in recent years. Numerous media platforms including The Sun, The Daily Mail and VICE promote this notion with a recent Sun headline in 2019: "Toxic shock horror: A tampon nearly killed me", with the article focusing primarily on the length of time the tampon was worn. According to Tampax, a tampon should be removed within four to eight, as indicated on the instructions in the box. Following public health awareness as a recognised association with "prolonged tampon use" and the development of TSS, there has been a decline in the incidence of TSS cases being reported in the following ten years Schuchat \& Broome [5].

With reference to tampon use, two requirements are needed for the development TSS. Firstly, the bacteria need an environment to multiply and grow and a tampon saturated in blood is a particularly supportive environment. Oxygen enables the bacteria to multiply and the space between the tampon fibres contributes to added air flow into the vagina resulting in a major site of staphylococcal growth and production Lina [6]. Secondly, bacteria entre the bloodstream through microscopic "tears" in the walls of the vagina caused by insertion or removal of the tampon. Bacteraemia may progress to potentially fatal septicaemia in some high-risk patients (e.g. younger adults).

Interestingly in this case, no tampon was retained at the time of the symptomatic presentation and on taking the history via the 
parents, it appears to have been removed at the appropriate time i.e. $<8$ hours. As TSS in menstruating women is associated with TSST-1 from Staphylococcus Aureus, the removal from the body and thus the removal of the toxin production poses further questioning behind the pathophysiology. In recent years, there has been a shift in the most prominent cause of TSS. Previously it was more commonly associated with menstrual factors, but studies have found a shift towards a higher association with non-menstrual causes; attributed to an increased public awareness and early recognition, a change in the tampon fibre composition and change in absorbance CDC [7].

However, some women continue to experience recurrent TSS due to menstrual causes and this can be found in one-third of patients with TSS Davis et al. [8]. In order for this to be possible, two conditions are essential: persistent colonization with a toxigenic strain of Staphylococcus Aureus and the continual lack of neutralizing antibody, found in patient with humoral immune response impairment Stolz et al. [9]. Such cases as these may provide an insight into why certain individuals are more susceptible to the development of menstrual TSS, especially in the absence of tampon use. Additional factors including age, anatomical variation and tampon brand have also been implicated in the susceptibility to develop TSS Schlech et al. [10]. Thus, there is a need for additional literacy on the pathophysiology, aetiology and management of TSS cases and this is a potential avenue for additional research.

From this current case, it is interesting to question that if the patient had presented with a "retained tampon", the diagnosis of Toxic Shock Syndrome would have been much quicker and ultimately the appropriate antibiotic regime could have been initiated much sooner. The primary aim of this report is to
DOI: $10.26717 /$ BJSTR.2019.20.003504

David D Souza. Biomed J Sci \& Tech Res

This work is licensed under Creative Commons Attribution 4.0 License

Submission Link: https://biomedres.us/submit-manuscript.php raise awareness among gynaecologists and other health care professionals that development of "menstrual associated" TSS can occur in the absence of a retained tampon. Such early recognition and treatment can promote better outcomes.

\section{Reference}

1. Pringle, P Raymond, Chung T (2018) Handbook of Liver Disease (Fourth edn.).

2. Ross A, Shoff HW (2019) Toxic Shock Syndrome. Treasure Island (FL): StatPearls Publishing.

3. Reingold AL, Hargrett NT, Shands KN, Dan BB, Schmid GP, et al. (1982) Toxic shock syndrome surveillance in the United States, 1980 to 1981. Annals of Internal Medicine 96: 875-880.

4. MacPhee RA, Miller WL, Gloor GB, McCormick JK, Hammond JA, et al. (2013) Influence of the vaginal microbiota on toxic shock syndrome toxin 1 production by Staphylococcus aureus. Applied and Environmental Microbiology 79(6): 1835-1842.

5. Schuchat, A, Broome CV (1991) Toxic shock syndrome and tampons. Epidemiology review 13: 99-112.

6. Lina G (2018) Tampons Can Cause Toxic Shock Syndrome.

7. (1990) Centres for Disease Control. Reduced incidence of menstrual toxic-shock syndrome: United States, 1980-1990, MMWR Morbidity Mortal Weekly Report 39(25): 421-423.

8. Davis JP, Osterholm MT, Gibson RW, Mandel JS, Wintermeyer LA, et al. (1982) Tri-state toxic shock syndrome study. II. Clinical and laboratory findings. Journal of Infectious Disease 145(4): 441-448.

9. Stolz SJ, Davis JP, Vergeront JM, Crass BA, Chesney PJ, et al. (1985) Development of serum antibody to toxic shock toxin among individuals with toxic shock syndrome in Wisconsin. Journal of Infectious Disease 151(5): 883-889.

10. Schlech WF, Shands KN, Reingold AL, Dan BB, Schmid GP, et al. (1982) Risk factors for development of toxic shock syndrome. Association with a tampon brand. JAMA 248(7): 835-839.

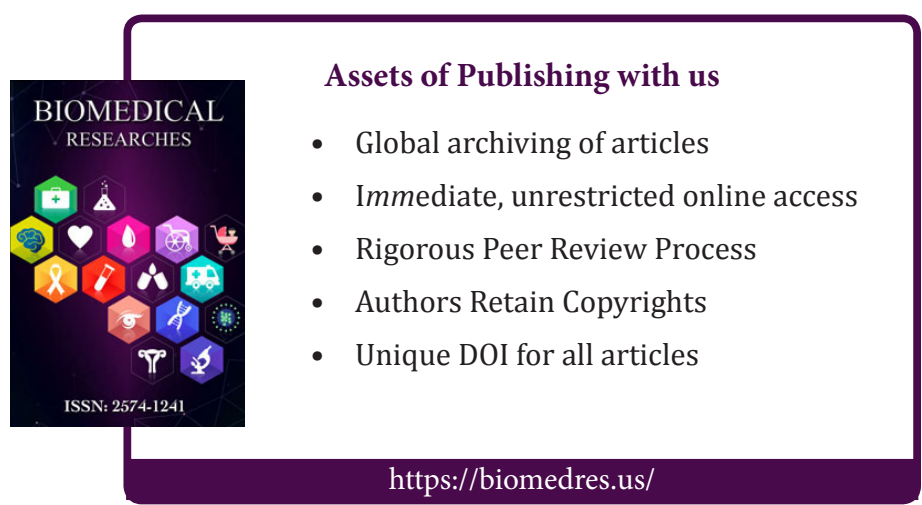

